\title{
Neurological Complications of Paroxysmal Nocturnal Hemoglobinuria
}

\author{
N. Jewel Samadder, Leanne Casaubon, Frank Silver, Rodrigo Cavalcanti
}

Can. J. Neurol. Sci. 2007; 34: 368-371

Paroxysmal nocturnal hemoglobinuria (PNH) is an acquired stem cell disorder characterized by three cardinal clinical manifestations: 1) hemolysis and hemoglobinuria, 2) thrombosis, and 3) bone marrow failure indistinguishable from aplastic anemia. ${ }^{1}$ Paroxysmal nocturnal hemoglobinuria is a rare disease with an incidence of 2-6 per million persons. Only 220 reported cases of PNH were found in one of the largest retrospective epidemiological studies in France from 1946 to $1995 .^{2}$

\section{Case Report}

A 54-year-old woman presented to the Emergency Department complaining of sudden onset of right-sided tinnitus followed by dizziness, dysarthria, nausea, and vomiting. Her only additional complaint was that of "cola" colored urine and dysuria. The patient denied a history of fever, seizures, focal weakness or sensory abnormalities. There was no history of cough, night sweats, weight loss or diarrhea. The patient's neurologic symptoms resolved within minutes, followed only by a self-limiting bi-frontal headache.

Her past medical history was significant for a diagnosis of paroxysmal nocturnal hemoglobinuria made at age 27 when she presented to hospital with symptoms of anemia. Investigations at that time, including a HAMs test, confirmed the diagnosis of PNH. After her initial management, she had not been followed by a haematologist.

Four years prior to the current admission she presented to hospital with a seizure and severe left hemiparesis. Imaging revealed a right frontal intracerebral haemorrhage (ICH) secondary to superior sagittal sinus thrombosis. She had full neurologic recovery from the $\mathrm{ICH}$, which was treated conservatively. She also had two non-ST-elevation myocardial infarctions (NSTEMI), the first in 2000 and the second was 20 days prior to her current hospitalization. The NSTEMI in 2000 had been treated conservatively with acetylsalicylic acid (ASA). The second NSTEMI in 2004 prompted an angiogram, which revealed an occlusive thrombus in the circumflex artery (see Figure 1). She was transferred to a tertiary care center for angioplasty after initial medical management but the second angiogram showed no further clot in the circumflex artery. Clopidogrel was added to ASA for secondary cardiac prevention. The patient had no other vascular risk factors including no history of hypertension, diabetes, or hyperlipidemia and she was a non-smoker.

With her current presentation, neurologic examination was completely normal. The general physical exam was also

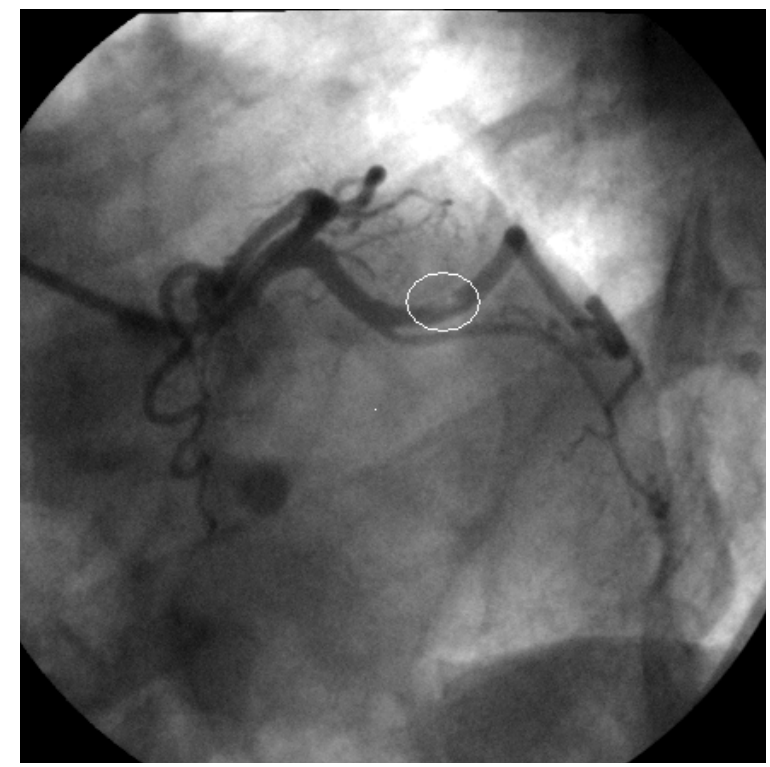

Figure 1. Cardiac catheterization (angiogram) showing occlusive thrombus in circumflex artery. The thrombus is indicated.

unremarkable. Her initial lab work was significant for hemoglobin $84 \mathrm{~g} / \mathrm{L}$ (mean corpuscle volume $96 \mathrm{fL}$ ), elevated bilirubin (total $43 \mathrm{uM}$ and indirect $34 \mathrm{uM}$ ), reticulocyte count $191 \mathrm{~g} / \mathrm{L}$, elevated AST $58 \mathrm{U} / \mathrm{L}$ and LDH $714 \mathrm{U} / \mathrm{L}$, and an undetectable haptoglobin $(<0.07 \mathrm{~g} / \mathrm{L})$. Electrolytes and fasting serum glucose were normal; creatinine was 46 . Peripheral blood film showed a normocytic anemia with no fragments consistent

From the Division of General Internal Medicine, Department of Medicine (NJS, RC), Division of Neurology, Department of Medicine (LC, FS), Toronto Western Hospital, University Health Network, Faculty of Medicine, University of Toronto, Toronto, ON; Division of Gastroenterology (NJS), University of Michigan Hospital, Ann Arbor, Michigan, USA.

ReCEIVED OCTOBER 4, 2006. ACCEPTED IN FINAL FORM APRIL 7, 2007.

Reprint requests to: N. Jewel Samadder, Division of Gastroenterology, University of Michigan Hospital, Ann Arbor, Michigan, 48109, USA. 


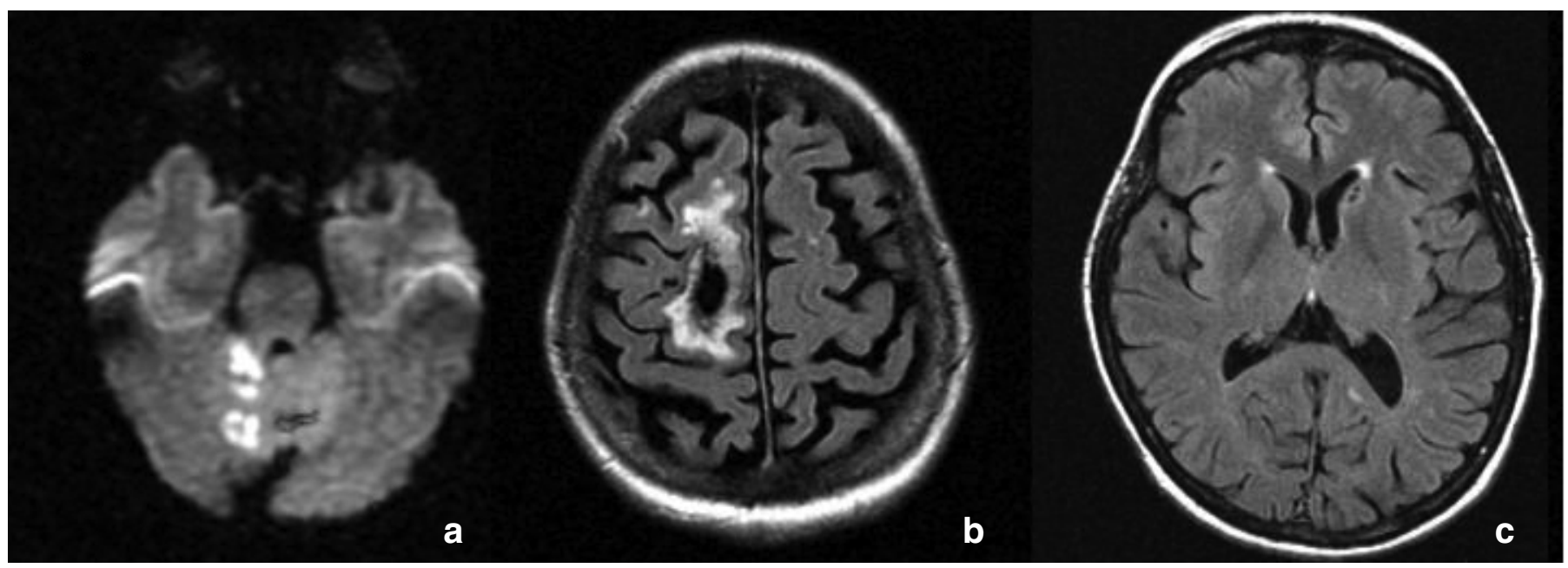

Figure 2. (a) MRI diffusion sequence. Acute ischemic stroke demonstrated by an area of restricted diffusion in the medial right cerebellar hemisphere. (b) MRI FLAIR sequence. Area of hyperintensity with a core of low signal in the right parasagittal frontal lobe from previous hemorrhage related to sagittal sinus thrombosis. Gradient echo (GRE) sequence (not shown) confirmed hemorrhage within the lesion. (c) MRI FLAIR sequence. Area of cystic change in the left caudate head indicating prior ischemic stroke.

with the laboratory studies for an intravascular haemolytic anemia. Brain CT showed no acute changes; an old right frontal parasagittal hemorrhage was seen.

Brain MRI, MR-angiogram (MRA) and MR-venogram (MRV) were done. The MRI revealed an acute right cerebellar infarct in the superior cerebellar artery (SCA) territory (see

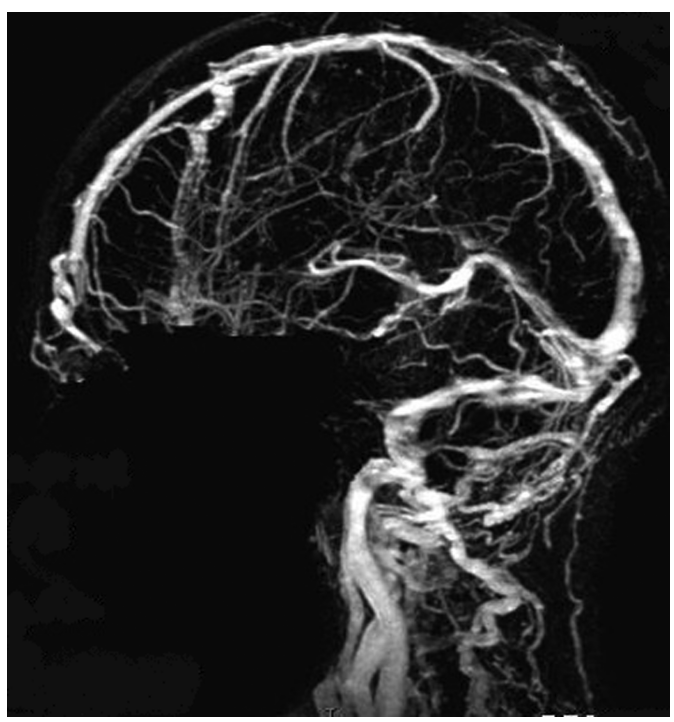

Figure 3. MR-Venogram (gadolinium enhanced protocol). Magnetic resonance venogram $(M R V)$ showing an area of focal narrowing of the superior sagittal sinus near the vertex consistent with chronic changes of a previous sinus thrombosis.
Figure 2a). Also noted were chronic changes of an old right frontal ICH as well as old infarcts in the left cerebellar hemisphere and left caudate head (see Figures $2 \mathrm{~b}$ and $2 \mathrm{c}$ ). The MRV demonstrated no acute venous thrombosis but showed features of a prior sagittal sinus thrombosis (see Figure 3 ). The MRA revealed no evidence of atherosclerotic disease within the carotid or vertebrobasilar vessels (not shown).

The patient's diagnosis of PNH was re-confirmed by flow cytometric analysis showing CD59 deficient erythrocytes of $74 \%$ and granulocytes of $79 \%$. Laboratory tests for other causes of hypercoagulability were negative (protein C $1.22 \mathrm{U} / \mathrm{ml}$, protein $\mathrm{S} 0.53 \mathrm{U} / \mathrm{ml}$, anti-thrombin III $1.0 \mathrm{U} / \mathrm{ml}$, homocysteine $8.8 \mathrm{uM}$, antiphospholipid antibody screen, prothrombin variant, and factor $\mathrm{V}$ Leiden). A transthoracic echocardiogram with saline bubble study was normal. Her fasting lipid profile was normal.

The patient had a transfusion of three units of packed red blood cells. For secondary stroke prevention she was continued on combined antiplatelet therapy with ASA and Clopidogrel but had a recurrence of neurological symptoms in hospital causing moderate left arm ataxia. She was then anticoagulated with unfractionated heparin, then coumadin. Low dose ASA was continued. Upon hospital discharge she had only mild residual incoordination in the left arm. Follow-up was arranged with hematology for consideration of eculizumab therapy or bone marrow transplant, the internal medicine ambulatory clinic, and the Stroke Prevention Clinic.

\section{Discussion}

Paroxysmal nocturnal hemoglobinuria is the only haemolytic anemia caused by an intrinsic defect that is acquired and not inherited. Defective stem cells arise from clonal expansion of a totipotent stem cell containing a somatic mutation in the 
phosphatidylinositol glycan complementation group A gene (PIG-A gene) on the X chromosome. ${ }^{3,4}$ More than 100 PIG-A mutations have now been described. ${ }^{5}$ The PIG-A gene produces the glycosylphosphatidylinositol (GPI) anchor that serves to attach a number of proteins to the cell membrane. ${ }^{6}$ Two such proteins, CD55 (Decay Accelerating Factor - DAF) and CD59 (Membrane Inhibitor of Reactive Lysis - MIRL), missing from the red blood cell surface in PNH, constitute the normal red cell's ability to resist complement-mediated lysis. ${ }^{7-11}$ Approximately $50 \%$ of PNH patients present with nocturnal hemoglobinuria. Darkening of the urine is most noticeable in the morning, either because the urine is more concentrated or there is increased hemolysis at night. ${ }^{12,13}$ Increased venous thrombotic episodes have been noted in patients with $\mathrm{PNH}$ and thrombosis related death occurs in $40 \%$ of patients. ${ }^{14,15}$ These thrombotic episodes can occur in any site throughout the body, however venous thrombosis has been reported more frequently than arterial. ${ }^{15,16}$ Classically, hepatic venous thrombosis (Budd-Chiari syndrome) has been associated with PNH. ${ }^{17,18}$ Arterial thrombosis has been reported rarely with involvement of cerebral, renal, splenic, iliac and coronary arteries, based on results of autopsy findings. ${ }^{19-21}$ The increased thrombosis is due to platelet activation via complement, an increase in circulating procoagulant activity resulting from erythrocyte hemolysis and release of adenosine diphosphate (ADP). ${ }^{10}$

Cerebral venous sinus thrombosis is seen in $\mathrm{PNH}$ and often carries a poor prognosis. ${ }^{16}$ Less frequently, arterial ischemic strokes occur within medium to large artery territories either in isolation or in patients with prior systemic venous thrombosis. ${ }^{20}$ At presentation, these patients are often younger than typical stroke patients and warrant complete work-up of rare stroke etiologies. These include hypercoagulable states (antiphospholipid antibodies, protein $\mathrm{C}$ or $\mathrm{S}$ deficiency, antithrombin III deficiency, prothrombin gene mutation, and MTHFR mutation), oral contraceptive use, paradoxical emboli related to an intra-cardiac shunt (patent foramen ovale or atrial septal defect), arterial dissection and primary central nervous system vasculitis. Atherosclerotic risk factors and cardiac arrhythmias must also be addressed if present. In some patients, a co-existent procoagulant factor is found to guide therapy, though in most cases PNH is the only risk factor. ${ }^{22,23}$ No randomized trials have evaluated the efficacy of antiplatelet agents or anticoagulation for primary or secondary prevention of stroke and sinovenous thrombosis in these patients.

A diagnosis of PNH should be entertained in all patients presenting with pancytopenia of unknown origin with reticulocytosis, non-immune hemolysis and venous thrombosis at an unusual anatomic site. Paroxysmal nocturnal hemoglobinuria can be differentiated from other haemolytic anemias by the presence of hemosiderinuria. Definitive diagnosis is established by the demonstration of GPI linked protein deficiencies on erythrocyte and neutrophil surfaces by flow cytometry.

Testing for PNH includes the sucrose-hemolysis test (HAMs test) based on complement-mediated lysis of erythrocytes in a low ionic-strength solution. ${ }^{5}$ Greater sensitivity and specificity may be achieved through the use of fluorescence activated cell sorter (FACS) to quantitate the percentage of cells deficient in CD59 or CD55. ${ }^{24}$ The percentage of cells completely deficient in
CD59 (Type III PNH cells) can be correlated with the degree and severity of clinical symptoms in PNH. ${ }^{5,24}$ Also, at least two GPI linked proteins (CD55 and CD59) should be analysed to exclude the rare inherited deficiencies of single antigens (selective CD55 deficiency). ${ }^{25,26}$

The only known cure for PNH is haematopoietic stem cell transplantation, which replaces the abnormal clone(s) present in the bone marrow. Supportive therapy includes red blood cell transfusion, which is believed to suppress marrow production of new PNH clones, thereby decreasing thrombotic complications. 5 ,13 Some evidence supports the use of anticoagulation for patients who have experienced a thrombotic event. A non-randomized study by Hall et $\mathrm{al}^{27}$ showed anticoagulation of patients with large $\mathrm{PNH}$ clones reduced the frequency of thrombotic events by $30 \%$. Iron and folic acid supplementation are recommended as in any haemolytic anemia. Recombinant tissue plasminogen activator (rtPA) may be used in the management of acute ischemic stroke following current guidelines for its use. Eculizumab, a recently developed complement inhibitor has entered clinical trials and has been shown to be safe and effective at reducing hemolysis in $\mathrm{PNH}$ patients. $^{28}$

The clinical course of PNH is quite variable. Patients with PNH may have a long-term chronic illness but the disease does shorten life. The median survival from diagnosis was 10 to 15 years in two large historical studies. ${ }^{2,15}$ Patients most commonly die of thrombosis or progressive cytopenias. Malignancy, such as myelodysplasia or acute myeloid leukemia supervenes in 2-3\% of cases. ${ }^{29}$ Due to a relatively hypercoaguable state, oral contraception and hormone replacement therapy should be avoided. Pregnancy can be considered with prophylactic anticoagulation in a high-risk obstetrical clinic. ${ }^{30}$

This case report provides a description of a rare hematologic disorder, PNH, its clinical presentation, pathophysiology, diagnostic tests and recommended treatment. The case also documents a rare neurologic presentation of $\mathrm{PNH}$ with both arterial and venous thrombosis.

\section{REFERENCES}

1. Rotoli B, Luzzatto I. Paroxysmal nocturnal hemoglobinuria. Baillierees Clin Haematol. 1989;2:13-38.

2. Socie G, Mary JY, de Gramont A, Rio B, Leporrier M, Rose C, et al. Paroxysmal nocturnal hemoglobinuria: long-term follow-up and prognostic factors. French Society of Hematology. Lancet. 1996;348:573-7.

3. Bessler M, Mason PJ, Hillmen P, Miyata T, Yamada N, Takeda J, et al. Paroxysmal nocturnal hemoglobinuria is caused by somatic mutations in the PIG-A gene. EMBO J. 1994;13:110-17.

4. Ware RE, Rosse WF, Howard TA. Mutations within the PIG-A gene in patients with paroxysmal nocturnal hemoglobinuria. Blood. 1994;83:2418-22.

5. Johnson RJ, Hillmen P. Paroxysmal nocturnal hemoglobinuria: nature's gene therapy? J Clin Pathol: Mol Pathol. 2002;55: 145-52.

6. Miyata T, Takeda J, Iida Y, Yamada N, Inoue N, Takahashi M, et al. The cloning of PIG-A, a component in the early step of GPIanchor biosynthesis. Science. 1993;259:18-20.

7. Holguin MH, Wilcox LA, Bernshaw NJ, Rossse WF, Parker CJ. Relationship between the membrane inhibitor of reactive lysis and the erythrocyte phenotypes of paroxysmal nocturnal hemoglobinuria. J Clin Invest. 1989;84:1387-94. 
8. Nicholson-Weller A, March JP, Rosenfeld SI, Susten KF. Affected erythrocytes of patients with paroxysmal nocturnal hemoglobinuria are deficient in the complement regulatory protein, decay accelerating factor. Proc Natl Acad Sci USA. 1983;80:5066-70.

9. Miyata T, Yamada N, Iida Y, Nishimura J, Takeda J, Kitani T, et al. Abnormalities of PIG-A transcripts in granulocytes from patients with paroxysmal nocturnal hemoglobinuria. N Engl J Med. 1994;330:249-55.

10. Rosse WF, Ware RE. The molecular basis of paroxysmal nocturnal hemoglobinurai. Blood. 1995;86:3277-86.

11. Rollins SA, Sims PJ. The complement inhibitory activity of CD59 resides in its capacity to block incorporation of $\mathrm{C} 9$ into membrane C5b-9. J Immunol. 1990;144:3478-83.

12. Ham GD, Horack HM. Chronic haemolytic anaemia with paroxysmal nocturnal hemoglobinuria. Arch Intern Med. 1941;67:115-36

13. Smith LJ. Paroxysmal nocturnal hemoglobinuria. Clin Lab Sci. 2004; $17: 172-7$

14. Peytremann RR, Hartmann RC. Thrombosis in paroxysmal nocturnal hemoglobinuria with particular reference to progressive, diffuse hepatic venous thrombosis. Set Haematol. 1972;3:115-36.

15. Hillmen P, Lewis SM, Bessler M, Luzzatto L, Dacie JV. Natural history of paroxysmal nocturnal hemoglobinura. N Engl J Med. 1995;333:1253-8.

16. Johnson RV, Kaplan SR, Blailock ZR. Cerebral venous thrombosis in paroxysmal nocturnal hemoglobinuria. Neurology. 1970;20:681-6.

17. Hartmann RC, Luter AB, Jenkins DR, Tenorio LE, Saba HI. Fulminant hepatic venous thrombosis (Budd-Chiari syndrome) in paroxysmal nocturnal hemoglobinuria: definition of medical emergency. Johns Hopkins Med J. 1980;146:247-54.

18. Valla D, Dhumeaus D, Babany G, Hillon P, Rueff B, Rochant H, et al. Hepatic vein thrombosis in paroxysmal nocturnal hemoglobinuria: a spectrum from asymptomatic occlusion of hepatic venules to fatal Budd-Chiari syndrome. Gastroenterology. 1987;93:569.
19. Klein KL, Hartmann RC. Acute coronary thrombosis in paroxysmal nocturnal hemoglobinuria. South Med J. 1989;82:1169-71.

20. Al-Samman MB, Cuetter AC. Cerebral arterial thrombosis as a complication of paroxysmal nocturnal hemoglobinuria. South Med J. 1994;87:1355-8.

21. Hyafil F, Montalescot G, Amoura Z, Choussat R, Collet JP, Piette JC. Recurrent myocardial infarction in a patient with paroxysmal nocturnal hemoglobinuria. Circulation. 2003;108: e91-e92.

22. Granata G, Izzo T, Di Micco P, Bonamassa B, Castaldo G, Viggiano VG, et al. Thromboembolic events and haematological diseases: a case of stroke as clinical onset of a paroxysmal nocturnal hemoglobinuria. Thrombosis Journal. 2004;2:10-14.

23. von Stuckrad-Barre S, Berkefeld J, Steckel D, Sitzer M. Cerebral arterial thrombosis in paroxysmal nocturnal hemoglobinuria. J Neurol. 2003;250:756-7.

24. Hall SE, Rosse WF. The use of monoclonal antibodies and flow cytometry in the diagnosis of paroxysmal nocturnal hemoglobinuria. Blood. 1996;87:5332-40.

25. Telen MJ, Green AM. The Inab phenotype: characterization of the membrane protein and complement regulatory defect. Blood. 1989;74:437-41.

26. Yamashina M, Ueda E, Kinoshita T, Takami T, Ojima A, Ono H, et al. Inherited complete deficiency of 20-kilodalton homologous restriction factor (CD59) as a cause of paroxysmal nocturnal hemoglobinuria. N Engl J Med. 1990;323:1184-9.

27. Hall C, Richards S, Hillmen P. Primary prophylaxsis with warfarin prevents thrombosis in paroxysmal nocturnal hemoglobinuria. Blood. 2003;102:3587-91.

28. Hillmen P, Hall C, Marsh JC, Elebute M, Bombara MP, Petro BE, et al. Effect of eculizumab on hemolysis and transfusion requirements in patients with paroxysmal nocturnal hemoglobinuria. N Engl J Med. 2004;350: 522-9.

29. Kinoshita T, Inoue N. Relationship between aplastic anemia and paroxysmal nocturnal hemoglobinuria. Int J Hematol. 2002; 75:117-22.

30. Bjorge L, Ernst P, Haram KO. Paroxysmal nocturnal hemoglobinuria in pregnancy. Acta Obstet Gynecol Sand. 2003; 82:1067-71. 